\title{
Synthesis of New Examples of Corands with 16-Membered P,N-Containing Core Ring
}

\author{
Andrey A. Karasik, ${ }^{a}$ Roman N. Naumov, $^{a}$ Kirill B. Kanunnikov, $^{a}$ \\ Dmitry B. Krivolapov, ${ }^{a}$ Igor A. Litvinov, ${ }^{a}$ Peter Lönnecke, ${ }^{b}$ Anna S. Balueva, ${ }^{a}{ }^{\circledR}$ \\ Elvira I. Musina, ${ }^{a}$ Evamarie Hey-Hawkins, $^{\mathrm{b}}$ and Oleg G. Sinyashin ${ }^{\mathrm{a}}$
}

Dedicated to Prof. Oscar Koifman on the occasion of his $70^{\text {th }}$ birthday

\author{
a'A.E. Arbuzov Institute of Organic and Physical Chemistry, Russian Academy of Sciences, Kazan Scientific Center, 420088 \\ Kazan, Russian Federation \\ ${ }^{\mathrm{b}}$ Institut für Anorganische Chemie der Universität Leipzig, 04103 Leipzig, Germany \\ ${ }^{\circledR}$ Corresponding authorE-mail: anna@iopc.ru
}

\begin{abstract}
An effective strategy of the synthesis of P,N-containing corands with 16-membered core ring is described. It is based on covalent self-assembly processes in the course of Mannich-type condensations in three component systems: bis(mesitylphosphino)propane-formaldehyde-primary amines. This approach allowed the authors to obtain a series of novel functionalized 1,9-di(aralkyl)-3,7,11,15-tetramesityl-1,9-diaza-3,7,11,15-tetraphosphacyclohexadecanes in very good yields. The study of structure of the synthesized corands showed that all of them were obtained as pure RSSRstereoisomers with similar conformations in the solid state.
\end{abstract}

Keywords: Macrocycle, phosphine, corand, covalent self-assembly.

\section{Синтез новых примеров корандов с 16-членным P,N-содержащим основным кольцом}

\author{
А. А. Карасик, ${ }^{a}$ P. Н. Наумов, ${ }_{1}{ }^{a}$ К. Б. Канунников, ${ }^{a}$ А. Б. Криволапов ${ }_{1}{ }^{a}$ И. А. Аитвинов, ${ }^{a}$ \\ П. леннекке, ${ }^{\mathrm{b}}$ А. С. Балуева, ${ }^{\mathrm{a}}$ Э. И. Мусина, ${ }^{a}$ Е. Хей-Хокинс, ${ }^{\mathrm{b}}$ О. Г. Синяшин ${ }^{\mathrm{a}}$
}

Посвящается член-корреспонденту РАН, профессору Оскару Иосифовичу Койфману в связи с 70-летием со дня рождения

\begin{abstract}
аФедеральное государственное бюджетное учреждение науки Институт органической и физической химии им. А.Е.Арбузова Казанского научного ичентра Российской академии наук, 420088 Казань, Российская Федерация ${ }^{\mathrm{b}}$ Институт неорганической химии Университета Лейпцฺига, 04103 Лейпичиг, Германия

@E-mail:anna@iopc.ru
\end{abstract}

Описана эффективная стратегия синтеза P,N-содержащих корандов с 16-членным основным кольцом, основанная на процессах ковалентной самосборки в ходе конденсации типа Манниха в трехкомпонентных системах: бис(мезитилфосфино)пропан-формальдегид-первичные амины. Данный подход позволил авторам получить серию новых функиионализированных 1,9-ди(аралкил)-3, 7,11,15-тетрамезитил-1,9-диаза-3, 7,11,15тетрафосфациклогексадеканов с очень хорошими выходами. Изучение структуры синтезированных корандов показало, что все они были получены в виде индивидуальных RSSR-стереоизомеров, имеющих сходные конформации в твердом состоянии.

Ключевые слова: Макроцикл, фосфин, коранд, ковалентная самосборка. 


\section{Introduction}

An ability of macrocycles to be highly selective receptors (host molecules) for a number of metal and organic cations or anions, as well as for the small or even huge (e.g. fullerenes) neutral organic substances is the reason of the interest to these compounds. ${ }^{[1-4]}$ So, macrocyclic chemistry plays an important role in the material science, especially in the construction of nano-sized materials on the "bottom-up" principles..$^{[1,25-7]}$ Incorporation of phosphorus atoms into the macrocyclic skeleton should lead to the specific properties of the discussed molecules. First of all $\mathrm{P}^{\mathrm{III}}$ donor centers are soft in contrast with the hard $\mathrm{O}$ and $\mathrm{N}$ counterparts, that is why the specific coordination ability to the soft metal ions should appear. The well-known ability of $\mathrm{P}^{\mathrm{III}}$ species to form transition metal based catalysts of various organic reactions should be modified by additional bonding of metal centers or organic substrates with macrocycle fragments via dative or weak interactions. In addition the specific reactivity and the structural variability of phosphorus could be utilized for the additional functionalization of the macrocyclic objects or for the rational design of macrocyclic molecular devices. ${ }^{[8,9]}$

However, there are some noticeable problems concerning the availability of P-based macrocycles. Along with the known instability of phosphines (the most intriguing class of ligands) to oxidation the cyclic compounds containing two or more phosphorus atoms are usually obtained as a mixture of stereoisomers due to the high inversion barrier of $\mathrm{P}^{\mathrm{III}}$ and these mixtures require the separation in order to obtain the individual macrocycles. So, the availability of the P-containing macrocyclic compounds is one of the key points for their use in the fields of catalysis, supramolecular chemistry and nanomaterials. At the present time three main strategies for the macrocyclic synthesis may be identified: 1) macrocyclization under high-dilution conditions; 2) template synthesis of macrocycles; 3) covalent self-assembly of macrocycles. The last approach, namely the covalent selfassembly of macrocycles, has been formed recently. The distinctive feature of the covalent self-assembly processes is their ability of the self-correction, when the "incorrect" intermediate or product is able to decompose into starting compounds due to the reversibility of the reaction. These compounds react further to give more thermodynamically stable "correct" combination. The self-correction is a reason of the high selectivity of the covalent self-assembly processes. ${ }^{[10-12]}$ The indication of self-assembly phenomenon is the high yield of macrocycles and the regio- and stereoselectivity of their formation in the course of the reversible reaction (often at elevated temperature) between two or more initial molecules (at least one of them must possess spatially divided functional groups) under conditions of relatively high concentration and without any templating reagents.

This synthetic strategy has been applied for the synthesis of $\mathrm{P}, \mathrm{N}$-containing macrocycles for the last 10 years and appeared to be rather effective. ${ }^{[13,14]}$ The Mannichtype condensation of primary and secondary phosphines as proton-donating reagents has been chosen as the basic reaction for the use of covalent self-assembly approach in the synthesis of P,N-containing macrocycles. Recently few examples of the new type of 16-membered P,Ncontaining macrocycles 1-4 namely 1,9-diaza-3,7,11,15tetraphosphacyclohexadecanes ${ }^{[13-16]}$ and first cryptand $\mathbf{5}$ with the same core ring ${ }^{[13,17]}$ (Scheme 1) have been described.

However, as it has been mentioned in the review of C.D. Swor, D.R. Tyler ${ }^{[18] ~ " t h i s ~ r o u t e ~ c a n n o t ~ y e t ~ b e ~ c o n s i d e r e d ~}$ a generalized method for the synthesis of macrocyclic phosphines". In the present paper we describe the synthesis and crystal structure investigations of several new corands with 1,9-diaza-3,7,11,15-tetraphosphacyclohexadecane core ring including macrocycles containing various functional substituents on nitrogen atoms and demonstrate the universality of this approach.

\section{Experimental}

\section{General}

${ }^{1} \mathrm{H}$ NMR spectra (BrukerAvance-DRX 400, $400 \mathrm{MHz}$; standard: $\mathrm{Me}_{4} \mathrm{Si}$ ). ${ }^{31} \mathrm{P}$ NMR spectra (BrukerAvance-DRX 400, $162 \mathrm{MHz}$; standard: external $\left.85 \% \mathrm{H}_{3} \mathrm{PO}_{4}\right) \cdot{ }^{13} \mathrm{C}$ NMR spectra

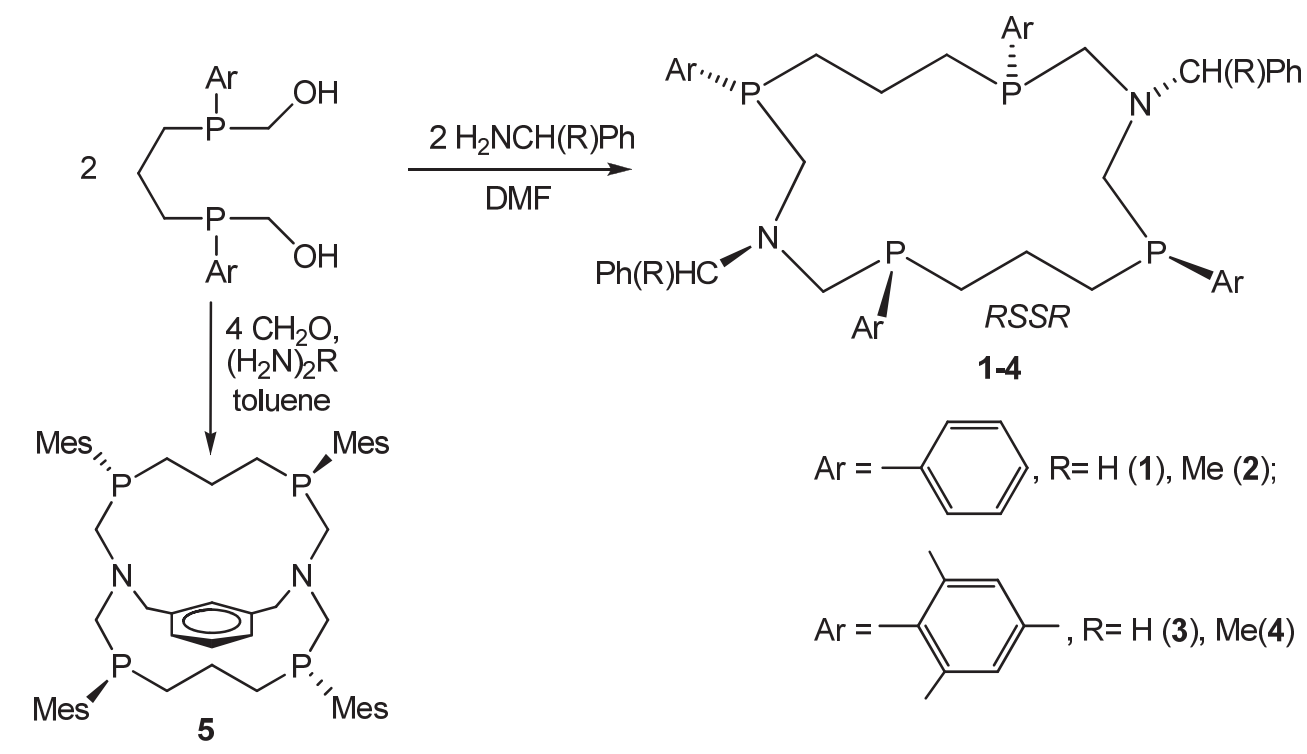

Scheme 1. 
(BrukerAvance-DRX 400, $100 \mathrm{MHz}$; standard: $\mathrm{Me}_{4} \mathrm{Si}$ ). ESI pos massspectra were obtained on Esquire 3000 plus mass-spectrometer. FAB mass spectra were obtained on a ZAB-HSQ-VG Analytical Manchester.The melting points were determined on a Boetius apparatus and are uncorrected.

1,3-Bis(mesitylphosphino)propane $\mathrm{e}^{[15,19]}$ was obtained according to the described method. All manipulations with the secondary phosphine and solutions of macrocycles were carried out by standard high-vacuum and dry-nitrogen techniques in dry degassed solvents which were purified by standard methods.

\section{Preparations}

(RSSR)-1,9-Di-(4'-methylbenzyl)-3,7,11,15-tetramesityl-1,9diaza-3,7,11,15-tetraphosphacyclohexadecane (6). A solution of 1,3-bis(mesitylphosphino)propane $(0.50 \mathrm{~g}, 1.45 \mathrm{mmol})$ and paraformaldehyde $(0.09 \mathrm{~g}, 3.0 \mathrm{mmol})$ in DMF $(5 \mathrm{~mL})$ was stirred at $80{ }^{\circ} \mathrm{C}$ for $1 \mathrm{~h}$ and a solution of 4-methylbenzylamine $(0.18 \mathrm{~g}$, $1.48 \mathrm{mmol})$ in DMF $(5 \mathrm{~mL})$ was added dropwise under stirring at $80^{\circ} \mathrm{C}$ for $3 \mathrm{~h}$. The reaction mixture was stirred at $80^{\circ} \mathrm{C}$ for 1 day. The resulting white crystals were collected by filtration, washed with DMF and dried carefully at 0.1 torr for $10 \mathrm{~h}$. Yield of 60.49 g (69 \%); m.p. $171-173{ }^{\circ} \mathrm{C}$. Found: C 76.30; H 8.70; N 3.21; P $12.18 \% . \mathrm{C}_{62} \mathrm{H}_{82} \mathrm{P}_{4} \mathrm{~N}_{2}$ [979.22] requires $\mathrm{C} 76.05 ; \mathrm{H}$ 8.44; N 2.86; $\mathrm{P}$ $12.65 \%$. ${ }^{1} \mathrm{H}$ NMR $\left(\mathrm{C}_{6} \mathrm{D}_{6}, 303 \mathrm{~K}\right) \delta_{\mathrm{H}} \mathrm{ppm}: 7.36\left(4 \mathrm{H}, \mathrm{d},{ }^{3} J_{H H}=7.5 \mathrm{~Hz}\right.$, $\left.\mathrm{C}^{13} \mathrm{H}\right), 7.11\left(4 \mathrm{H}, \mathrm{d},{ }^{3} J_{H H}=7.5 \mathrm{~Hz}, \mathrm{C}^{12} \mathrm{H}\right), 6.75\left(8 \mathrm{H}, \mathrm{s}, \mathrm{C}^{6} \mathrm{H}\right), 4.63(4 \mathrm{H}$, $\left.\mathrm{d},{ }^{2} J_{H H}=12.4 \mathrm{~Hz}, \mathrm{C}^{1} \mathrm{H}_{\mathrm{B}}\right), 4.21\left(2 \mathrm{H}\right.$, br.d, $\left.{ }^{2} J_{H H}=13.2 \mathrm{~Hz}, \mathrm{C}^{10} \mathrm{H}_{\mathrm{B}}\right), 3.16$ $\left(2 \mathrm{H}, \mathrm{d},{ }^{2} J_{H H}=13.2 \mathrm{~Hz}, \mathrm{C}^{10} \mathrm{H}_{\mathrm{A}}\right), 2.69\left(4 \mathrm{H}, \mathrm{dd},{ }^{2} J_{H H}=12.4 \mathrm{~Hz},{ }^{2} J_{H P}=9.91\right.$ $\left.\mathrm{Hz}, \mathrm{C}^{1} \mathrm{H}_{\mathrm{A}}\right), 2.59\left(24 \mathrm{H}, \mathrm{s}, \mathrm{C}^{8} \mathrm{H}\right), 2.42\left(4 \mathrm{H}, \mathrm{m}, \mathrm{C}^{2} \mathrm{H}\right), 2.28(2 \mathrm{H}, \mathrm{m}$, $\left.\mathrm{C}^{2} \mathrm{H}\right), 2.17\left(6 \mathrm{H}, \mathrm{s}, \mathrm{C}^{15} \mathrm{H}\right), 2.09\left(12 \mathrm{H}, \mathrm{s}, \mathrm{C}^{9} \mathrm{H}\right), 1.97\left(4 \mathrm{H}, \mathrm{m}, \mathrm{C}^{2} \mathrm{H}+\right.$ $\left.\mathrm{C}^{3} \mathrm{H}\right), 1.82\left(2 \mathrm{H}, \mathrm{m}, \mathrm{C}^{3} \mathrm{H}\right) .{ }^{13} \mathrm{C}$ NMR $\left(\mathrm{CDCl}_{3}, 303 \mathrm{~K}\right) \delta_{\mathrm{C}}$ ppm: 144.53 $\left(\mathrm{d},{ }^{2} J_{C P}=15.3 \mathrm{~Hz}, \mathrm{C}^{5}\right), 138.69\left(\mathrm{~s}, \mathrm{C}^{7}\right), 136.70\left(\mathrm{~s}, \mathrm{C}^{11}\right), 136.17(\mathrm{~s}$, $\left.\mathrm{C}^{14}\right), 130.89\left(\mathrm{~d},{ }^{1} J_{C P}=20.9 \mathrm{~Hz}, \mathrm{C}^{4}\right), 128.07-130.24\left(\mathrm{~m}, \mathrm{C}^{6}+\mathrm{C}^{12}+\mathrm{C}^{13}\right)$, 55.43-59.99 (br.m, $\left.\mathrm{C}^{1}+\mathrm{C}^{10}\right), 23.58\left(\mathrm{~d},{ }^{3} J_{C P}=18.7 \mathrm{~Hz}, \mathrm{C}^{8}\right), 20.21-$ $29.11\left(\mathrm{~m}, \mathrm{C}^{2}+\mathrm{C}^{3}+\mathrm{C}^{15}\right), 20.88\left(\mathrm{~d},{ }^{5} J_{C P}=10.2 \mathrm{~Hz}, \mathrm{C}^{9}\right) .{ }^{31} \mathrm{P} \mathrm{NMR}\left(\mathrm{C}_{6} \mathrm{D}_{6}\right.$, $303 \mathrm{~K}) \delta_{\mathrm{p}}$ ppm: $-41.70(\mathrm{~s})$.

(RSSR)-1,9-Di-(4'-tert-butylbenzyl)-3,7,11,15-tetramesityl1,9-diaza-3,7,11,15-tetraphosphacyclohexadecane (7) was obtained like 6 from 1,3-bis(mesitylphosphino)propane ( $0.50 \mathrm{~g}, 1.45$ $\mathrm{mmol})$, paraformaldehyde $(0.09 \mathrm{~g}, 3.0 \mathrm{mmol})$ and 4-tert-butylbenzylamine (0.24 g, $1.47 \mathrm{mmol})$. Yield of $70.50 \mathrm{~g} \mathrm{(65 \% );} \mathrm{m.p.} \mathrm{191-}$ $193{ }^{\circ}$ C. Found: C 76.50; H 8.62; N 3.11; P $11.58 \%$. $\mathrm{C}_{68} \mathrm{H}_{94} \mathrm{P}_{4} \mathrm{~N}_{2}$ [1063.38] requires C 76.80; H 8.91; N 2.63; P $11.65 \%$. ${ }^{1} \mathrm{H}$ NMR $\left(\mathrm{C}_{6} \mathrm{D}_{6}, 303 \mathrm{~K}\right) \delta_{\mathrm{H}}$ ppm: $7.39\left(4 \mathrm{H}, \mathrm{d},{ }^{3} \mathrm{~J}_{H H}=8.8 \mathrm{~Hz}, \mathrm{C}^{13} \mathrm{H}\right), 7.35(4 \mathrm{H}$, $\left.\mathrm{d},{ }^{3} J_{H H}=8.8 \mathrm{~Hz}, \mathrm{C}^{12} \mathrm{H}\right), 6.74\left(8 \mathrm{H}, \mathrm{s}, \mathrm{C}^{6} \mathrm{H}\right), 4.60\left(4 \mathrm{H}, \mathrm{d},{ }^{2} J_{H H}=12.4\right.$ $\left.\mathrm{Hz}, \mathrm{C}^{1} \mathrm{H}_{\mathrm{B}}\right), 4.19\left(2 \mathrm{H}\right.$, br.d, $\left.{ }^{2} J_{H H}=13.2 \mathrm{~Hz}, \mathrm{C}^{10} \mathrm{H}_{\mathrm{B}}\right), 3.24(2 \mathrm{H}, \mathrm{d}$, $\left.{ }^{2} J_{H H}=13.2 \mathrm{~Hz}, \mathrm{C}^{10} \mathrm{H}_{\mathrm{A}}\right), 2.76\left(4 \mathrm{H}, \mathrm{m}, \mathrm{C}^{1} \mathrm{H}_{\mathrm{A}}\right), 2.58\left(24 \mathrm{H}, \mathrm{s}, \mathrm{C}^{8} \mathrm{H}\right)$, $2.08\left(12 \mathrm{H}, \mathrm{s}, \mathrm{C}^{9} \mathrm{H}\right), 1.94-2.41\left(12 \mathrm{H}, \mathrm{m}, \mathrm{C}^{2} \mathrm{H}+\mathrm{C}^{3} \mathrm{H}\right), 1.29(18 \mathrm{H}, \mathrm{s}$, $\left.\mathrm{C}^{16} \mathrm{H}\right) \cdot{ }^{13} \mathrm{C}$ NMR $\left(\mathrm{CDCl}_{3}, 303 \mathrm{~K}\right) \delta_{\mathrm{C}} \mathrm{ppm}: 149.56\left(\mathrm{~s}, \mathrm{C}^{14}\right), 144.52$ $\left(\mathrm{d},{ }^{2} J_{C P}=15.0 \mathrm{~Hz}, \mathrm{C}^{5}\right), 138.66\left(\mathrm{~s}, \mathrm{C}^{7}\right), 136.63 \mathrm{c} .\left(\mathrm{s}, \mathrm{C}^{11}\right), 130.92$ $\left(\mathrm{d},{ }^{1} J_{C P}=21.2 \mathrm{~Hz}, \mathrm{C}^{4}\right), 128.38-129.90\left(\mathrm{~m}, \mathrm{C}^{6}+\mathrm{C}^{12}+\mathrm{C}^{13}\right), 55.45-59.99$ (br.m, $\mathrm{C}^{1}+\mathrm{C}^{10}$ ), $34.41\left(\mathrm{~s}, \mathrm{C}^{16}\right), 28.20-32.10\left(\mathrm{~m}, \mathrm{C}^{2}+\mathrm{C}^{3}+\mathrm{C}^{15}\right), 23.55$ $\left(\mathrm{d},{ }^{3} J_{C P}=18.7 \mathrm{~Hz}, \mathrm{C}^{8}\right), 20.87\left(\mathrm{~d},{ }^{5} J_{C P}=10.2 \mathrm{~Hz}, \mathrm{C}^{9}\right) .{ }^{31} \mathrm{P}$ NMR $\left(\mathrm{C}_{6} \mathrm{D}_{6}\right.$, $303 \mathrm{~K}) \delta_{\mathrm{p}}$ ppm: -41.70 (s).

(RSSR)-1,9-Di-(2'-phenylethyl)-3,7,11,15-tetramesityl-1,9diaza-3,7,11,15-tetraphosphacyclohexadecane (8) was obtained like 6 from 1,3-bis(mesitylphosphino)propane $(0.20 \mathrm{~g}, 0.58 \mathrm{mmol})$, paraformaldehyde $(0.034 \mathrm{~g}, 1.16 \mathrm{mmol})$ and 2-phenylethylamine (0.07 g, $0.57 \mathrm{mmol})$. Yield of $80.13 \mathrm{~g}(47 \%)$; m.p. $168-170{ }^{\circ} \mathrm{C}$. Found: C 75.81; H 8.31; N 3.20; P $12.47 \%$. $\mathrm{C}_{62} \mathrm{H}_{82} \mathrm{P}_{4} \mathrm{~N}_{2}$ [979.22] requires $\mathrm{C} 76.05 ; \mathrm{H}$ 8.44; $\mathrm{N}$ 2.86; P $12.65 \%$. $\mathrm{m} / z$ (ESI $)(\%): 979.6$ (100) $[\mathrm{M}+\mathrm{H}]^{+} .{ }^{1} \mathrm{H}$ NMR $\left(\mathrm{C}_{6} \mathrm{D}_{6}, 303 \mathrm{~K}\right) \delta_{\mathrm{H}} \mathrm{ppm}$ : 7.03-7.21 $(10 \mathrm{H}, \mathrm{m}$, $\left.\mathrm{C}^{13} \mathrm{H}+\mathrm{C}^{14} \mathrm{H}+\mathrm{C}^{15} \mathrm{H}\right), 6.78\left(8 \mathrm{H}, \mathrm{s}, \mathrm{C}^{6} \mathrm{H}\right), 4.11\left(4 \mathrm{H}, \mathrm{d},{ }^{2} J_{H H}=13.0 \mathrm{~Hz}\right.$, $\left.\mathrm{C}^{1} \mathrm{H}_{\mathrm{B}}\right), 3.27-3.44\left(2 \mathrm{H}, \mathrm{m}, \mathrm{C}^{10} \mathrm{H}_{\mathrm{B}}\right), 2.73-2.91\left(8 \mathrm{H}, \mathrm{m}, \mathrm{C}^{10} \mathrm{H}_{\mathrm{A}}+\mathrm{C}^{1} \mathrm{H}_{+}+\right.$ $\left.\mathrm{C}^{11} \mathrm{H}\right), 2.63-2.73\left(4 \mathrm{H}, \mathrm{m}, \mathrm{C}^{11} \mathrm{H}+\mathrm{C}^{2} \mathrm{H}\right), 2.61\left(24 \mathrm{H}, \mathrm{s}, \mathrm{C}^{8} \mathrm{H}\right), 2.41-2.54$ $\left(4 \mathrm{H}, \mathrm{m}, \mathrm{C}^{2} \mathrm{H}\right), 2.16-2.27\left(2 \mathrm{H}, \mathrm{m}, \mathrm{C}^{2} \mathrm{H}\right), 2.08\left(12 \mathrm{H}, \mathrm{s}, \mathrm{C}^{9} \mathrm{H}\right), 1.99-$ $2.08\left(2 \mathrm{H}, \mathrm{m}, \mathrm{C}^{3} \mathrm{H}\right), 1.78-1.95\left(2 \mathrm{H}, \mathrm{m}, \mathrm{C}^{3} \mathrm{H}\right) .{ }^{31} \mathrm{P}$ NMR $\left(\mathrm{C}_{6} \mathrm{D}, 303\right.$ K) $\delta_{\mathrm{p}}$ ppm: $-43.84(\mathrm{~s})$.
(RSSR)-1,9-Di-(3'-phenylprop-1 '-yl)-3, 7,11,15-tetramesityl1,9-diaza-3, 7,11,15-tetraphosphacyclohexadecane (9) was obtained like 6 from 1,3-bis(mesitylphosphino)propane ( $0.40 \mathrm{~g}, 1.16 \mathrm{mmol})$, paraformaldehyde $(0.08 \mathrm{~g}, 2.6 \mathrm{mmol})$ and 3-phenylprop-1-ylamine (0.15 g, $1.11 \mathrm{mmol})$. Yield of $90.35 \mathrm{~g}(61 \%)$; m.p. 173-175 ${ }^{\circ} \mathrm{C}$. Found: $\mathrm{C} 76.03 ; \mathrm{H} 8.82 ; \mathrm{N} \mathrm{3.15}$; P $12.08 \% . \mathrm{C}_{64} \mathrm{H}_{86} \mathrm{P}_{4} \mathrm{~N}_{2}[1007.28]$ requires $\mathrm{C} 76.31 ; \mathrm{H} 8.61 ; \mathrm{N} 2.78 ; \mathrm{P} 12.30 \%$. ${ }^{1} \mathrm{H}$ NMR $\left(\mathrm{C}_{6} \mathrm{D}_{6}, 303\right.$ K) $\delta_{\mathrm{H}} \mathrm{ppm}: \quad 7.07-7.22\left(10 \mathrm{H}, \mathrm{m}, \mathrm{C}^{13} \mathrm{H}+\mathrm{C}^{14} \mathrm{H}+\mathrm{C}^{15} \mathrm{H}\right), 6.75(8 \mathrm{H}, \mathrm{s}$, $\left.\mathrm{C}^{6} \mathrm{H}\right), 4.08\left(4 \mathrm{H}\right.$, br.d, $\left.{ }^{2} J_{H H}=13.2 \mathrm{~Hz}, \mathrm{C}^{1} \mathrm{H}_{\mathrm{B}}\right), 3.11\left(2 \mathrm{H}, \mathrm{m}, \mathrm{C}^{10} \mathrm{H}_{\mathrm{B}}\right)$, $2.71\left(2 \mathrm{H}, \mathrm{m}, \mathrm{C}^{10} \mathrm{H}_{\mathrm{A}}\right), 2.58\left(24 \mathrm{H}, \mathrm{s}, \mathrm{C}^{8} \mathrm{H}\right), 2.12-2.67\left(14 \mathrm{H}, \mathrm{m}, \mathrm{C}^{2} \mathrm{H}+\right.$ $\left.\mathrm{C}^{1} \mathrm{H}_{\mathrm{A}}+\mathrm{C}^{12} \mathrm{H}\right), 2.07\left(12 \mathrm{H}, \mathrm{s}, \mathrm{C}^{9} \mathrm{H}\right), 1.61-1.93\left(10 \mathrm{H}, \mathrm{m}, \mathrm{C}^{2} \mathrm{H}+\mathrm{C}^{3} \mathrm{H}+\right.$ $\left.\mathrm{C}^{11} \mathrm{H}\right) .{ }^{13} \mathrm{C}$ NMR $\left(\mathrm{CDCl}_{3}, 303 \mathrm{~K}\right) \delta_{\mathrm{C}} \mathrm{ppm}: 149.53\left(\mathrm{~s}, \mathrm{C}^{16}\right), 144.52$ $\left(\mathrm{d},{ }^{2} J_{C P}=15.0 \mathrm{~Hz}, \mathrm{C}^{5}\right), 138.67\left(\mathrm{~s}, \mathrm{C}^{7}\right), 136.85\left(\mathrm{~s}, \mathrm{C}^{13}\right), 130.90$ $\left(\mathrm{d},{ }^{1} J_{C P}=21.2 \mathrm{~Hz}, \mathrm{C}^{4}\right), 128.30-129.60\left(\mathrm{~m}, \mathrm{C}^{6}+\mathrm{C}^{14}+\mathrm{C}^{15}\right)$, 54.9560.65 (br.m, $\left.\mathrm{C}^{1}+\mathrm{C}^{10}\right), 28.28-32.12\left(\mathrm{~m}, \mathrm{C}^{2}+\mathrm{C}^{3}+\mathrm{C}^{11}+\mathrm{C}^{12}\right), 23.51(\mathrm{~d}$, $\left.{ }^{3} J_{C P}=18.8 \mathrm{~Hz}, \mathrm{C}^{8}\right), 20.80\left(\mathrm{~d},{ }^{5} J_{C P}=10.1 \mathrm{~Hz}, \mathrm{C}^{9}\right) .{ }^{31} \mathrm{P} \mathrm{NMR}\left(\mathrm{C}_{6} \mathrm{D}_{6}, 303\right.$ K) $\delta_{\mathrm{p}}$ ppm: $-41.50(\mathrm{~s})$.

(RSSR)-1,9-Di-[(benzo[d][1",3"]dioxol-4'-yl)methyl]3,7,11,15-tetramesityl-1,9-diaza-3,7,11,15-tetraphosphacyclohexadecane (10) was obtained like $\mathbf{6}$ from 1,3bis(mesitylphosphino)propane $\quad(0.30 \quad \mathrm{~g}, \quad 0.87 \quad \mathrm{mmol})$, paraformaldehyde $(0.05 \mathrm{~g}, 1.67 \mathrm{mmol})$ and (benzo[d][1',3']dioxol4-yl)methylamine $(0.13 \mathrm{~g}, 0.86 \mathrm{mmol})$. Yield of $\mathbf{1 0} 0.15 \mathrm{~g}(33$ \%); m.p. $164-166{ }^{\circ}$ C. Found: C 71.93; H 7.78; N 2.96; P $11.67 \%$. $\mathrm{C}_{62} \mathrm{H}_{78} \mathrm{P}_{4} \mathrm{~N}_{2} \mathrm{O}_{4}[1039.19]$ requires $\mathrm{C} 71.66 ; \mathrm{H}$ 7.57; N 2.70; $\mathrm{P} 11.92$ \%. ${ }^{1} \mathrm{H}$ NMR $\left(\mathrm{C}_{6} \mathrm{D}_{6}, 303 \mathrm{~K}\right) \delta_{\mathrm{H}} \mathrm{ppm}: 6.98\left(2 \mathrm{H}, \mathrm{s}, \mathrm{C}^{13} \mathrm{H}\right), 6.75(\mathrm{~s}$, $\left.\mathrm{C}^{6} \mathrm{H}\right)$ 6.78-6.70 (m, $\left.\mathrm{C}^{12} \mathrm{H}+\mathrm{C}^{14} \mathrm{H}\right)$ (total intensity $\left.12 \mathrm{H}\right), 5.38(4 \mathrm{H}$, br.s, $\left.\mathrm{C}^{17} \mathrm{H}\right), 4.52\left(2 \mathrm{H}, \mathrm{d},{ }^{2} J_{H H}=12.9 \mathrm{~Hz}, \mathrm{C}^{10} \mathrm{H}_{\mathrm{B}}\right), 4.20\left(4 \mathrm{H}, \mathrm{d},{ }^{2} J_{H H}=\right.$ $\left.12.8 \mathrm{~Hz}, \mathrm{C}^{1} \mathrm{H}_{\mathrm{B}}\right), 3.07\left(2 \mathrm{H}, \mathrm{d},{ }^{2} J_{H H}=12.9 \mathrm{~Hz}, \mathrm{C}^{10} \mathrm{H}_{\mathrm{A}}\right), 2.71(4 \mathrm{H}, \mathrm{dd}$ $\left.{ }^{2} J_{H H}=12.8 \mathrm{~Hz},{ }^{2} J_{P H}=8.8 \mathrm{~Hz}, \mathrm{C}^{1} \mathrm{H}_{\mathrm{A}}\right), 2.59\left(24 \mathrm{H}, \mathrm{s}, \mathrm{C}^{8} \mathrm{H}\right), 2.49-2.35$ $\left(4 \mathrm{H}, \mathrm{m}, \mathrm{C}^{2} \mathrm{H}\right), 2.32-2.17\left(2 \mathrm{H}, \mathrm{m}, \mathrm{C}^{2} \mathrm{H}\right), 2.09\left(12 \mathrm{H}, \mathrm{s}, \mathrm{C}^{9} \mathrm{H}\right), 2.04-1.91$ $\left(4 \mathrm{H}, \mathrm{m}, \mathrm{C}^{2} \mathrm{H}+\mathrm{C}^{3} \mathrm{H}\right), 1.92-1.76\left(2 \mathrm{H}, \mathrm{m}, \mathrm{C}^{3} \mathrm{H}\right) .{ }^{31} \mathrm{P}$ NMR $\left(\mathrm{C}_{6} \mathrm{D}_{6}, 303\right.$ K) $\delta_{\mathrm{P}}$ ppm: $-44.29(\mathrm{~s})$.

(RSSR)-1,9-Di-[(pyridine-2'-yl)methyl]-3,7,11,15tetramesityl-1,9-diaza-3, 7,11,15-tetraphosphacyclohexadecane (11) was obtained like 6 from 1,3-bis(mesitylphosphino)propane (1.64 g, $4.76 \mathrm{mmol})$, paraformaldehyde $(0.29 \mathrm{~g}, 9.67 \mathrm{mmol})$ and 2-(aminomethyl)pyridine (0.52 g, $4.81 \mathrm{mmol})$. Yield of 111.64 g (72 \%); m.p. $187-189{ }^{\circ} \mathrm{C}$. Found: C 72.84; H 8.30; N 5.71; P $12.91 \% . \mathrm{C}_{58} \mathrm{H}_{76} \mathrm{P}_{4} \mathrm{~N}_{4}[953.15]$ requires $\mathrm{C} 73.09 ; \mathrm{H}$ 8.04; $\mathrm{N}$ 5.88; $\mathrm{P}$ $13.00 \% . \mathrm{m} / z$ (FAB) (\%): 883 (13.5) [M-CH $\left.\mathrm{CH}_{2} \mathrm{Py}+\mathrm{Na}\right]^{+}, 1015$ (4.3) $[\mathrm{M}+\mathrm{Na}+\mathrm{K}]^{+} .{ }^{1} \mathrm{H}$ NMR $\left(\mathrm{C}_{6} \mathrm{D}, 303 \mathrm{~K}\right) \delta_{\mathrm{H}} \mathrm{ppm}: 8.46\left(2 \mathrm{H}, \mathrm{m}, \mathrm{C}^{15} \mathrm{H}\right)$, $7.55\left(2 \mathrm{H}, \mathrm{d},{ }^{3} \mathrm{~J}_{H H}=7.3 \mathrm{~Hz}, \mathrm{C}^{12} \mathrm{H}\right), 7.31\left(2 \mathrm{H}, \mathrm{dd},{ }^{3} J_{H H} \approx^{3} J_{H H} \approx 7.3 \mathrm{~Hz}\right.$, $\left.\mathrm{C}^{13} \mathrm{H}\right), 6.74\left(8 \mathrm{H}, \mathrm{s}, \mathrm{C}^{6} \mathrm{H}\right), 6.73\left(2 \mathrm{H}, \mathrm{m}, \mathrm{C}^{14} \mathrm{H}\right), 4.75(4 \mathrm{H}$, br.d, $\left.{ }^{2} J_{H H}=12.4 \mathrm{~Hz}, \mathrm{C}^{1} \mathrm{H}_{\mathrm{B}}\right), 4.21\left(2 \mathrm{H}\right.$, br.d, $\left.{ }^{2} J_{H H}=13.2 \mathrm{~Hz}, \mathrm{C}^{10} \mathrm{H}_{\mathrm{B}}\right), 3.71$ $\left(2 \mathrm{H}\right.$, br.d, $\left.{ }^{2} J_{H H}=13.2 \mathrm{~Hz}, \mathrm{C}^{10} \mathrm{H}_{\mathrm{A}}\right), 2.76\left(4 \mathrm{H}, \mathrm{dm},{ }^{2} J_{H H}=12.4 \mathrm{~Hz}\right.$, $\left.\mathrm{C}^{1} \mathrm{H}_{\mathrm{A}}\right), 2.55\left(24 \mathrm{H}, \mathrm{s}, \mathrm{C}^{8} \mathrm{H}\right), 2.08\left(12 \mathrm{H}, \mathrm{s}, \mathrm{C}^{9} \mathrm{H}\right), 1.92-2.37(12 \mathrm{H}$, $\left.\mathrm{m}, \mathrm{C}^{2} \mathrm{H}+\mathrm{C}^{3} \mathrm{H}\right) \cdot{ }^{13} \mathrm{C}$ NMR $\left(\mathrm{CDCl}_{3}, 303 \mathrm{~K}\right) \delta_{\mathrm{C}} \mathrm{ppm}: 149.56(\mathrm{~s}$, $\left.\mathrm{C}^{15}+\mathrm{C}^{11}\right), 144.33\left(\mathrm{~d},{ }^{2} J_{C P}=15.0 \mathrm{~Hz}, \mathrm{C}^{5}\right), 138.66\left(\mathrm{~s}, \mathrm{C}^{7}\right), 136.63(\mathrm{~s}$, $\left.\mathrm{C}^{13}\right), 130.92\left(\mathrm{~d},{ }^{1} J_{C P}=21.2 \mathrm{~Hz}, \mathrm{C}^{4}\right), 121.27-123.55\left(\mathrm{~m}, \mathrm{C}^{6}+\mathrm{C}^{12}+\mathrm{C}^{14}\right)$, 55.43-59.99 (br.m, $\left.\mathrm{C}^{1}+\mathrm{C}^{10}\right), 28.08-29.53\left(\mathrm{~m}, \mathrm{C}^{2}+\mathrm{C}^{3}\right), 23.55$ (d, $\left.{ }^{3} J_{C P}=19.3 \mathrm{~Hz}, \mathrm{C}^{8}\right), 20.87\left(\mathrm{~s}, \mathrm{C}^{9}\right) .{ }^{31} \mathrm{P}$ NMR $\left(\mathrm{C}_{6} \mathrm{D}_{6}, 303 \mathrm{~K}\right) \delta_{\mathrm{p}} \mathrm{ppm}$ : $-41.30(\mathrm{~s})$.

(RSSR)-1,9-Di-[(pyridine-3'-yl)methyl]-3, 7,11,15tetramesityl-1,9-diaza-3, 7,11,15-tetraphosphacyclohexadecane (12). A solution of 1,3-bis(mesitylphosphino)propane (1.22 g, 3.54 $\mathrm{mmol})$ and paraformaldehyde $(0.21 \mathrm{~g}, 7.0 \mathrm{mmol})$ in toluene $(5 \mathrm{~mL})$ was stirred at $50{ }^{\circ} \mathrm{C}$ for $1 \mathrm{~h}$ and a solution of 3-(aminomethyl)pyridine $(0.38 \mathrm{~g}, 3.51 \mathrm{mmol})$ in toluene $(25 \mathrm{~mL})$ was added dropwise under stirring at $75{ }^{\circ} \mathrm{C}$ for $3 \mathrm{~h}$. The reaction mixture was stirred at $75{ }^{\circ} \mathrm{C}$ for 1 day. The resulting white crystals were collected by filtration, washed with toluene and dried carefully at 0.1 torr for 10 h. Yield of $120.95 \mathrm{~g}(56 \%)$; m.p. $174-176{ }^{\circ} \mathrm{C}$. Found: C 72.70; H 8.32; N 6.20; $\mathrm{P} 12.64 \% . \mathrm{C}_{58} \mathrm{H}_{76} \mathrm{P}_{4} \mathrm{~N}_{4}[953.15]$ requires $\mathrm{C} 73.09 ; \mathrm{H}$ 8.04; N 5.88; P $13.00 \%$. ${ }^{1} \mathrm{H}$ NMR $\left(\mathrm{C}_{6} \mathrm{D}_{6}, 303 \mathrm{~K}\right) \delta_{\mathrm{H}} \mathrm{ppm}: 8.59(2 \mathrm{H}$ $\left.\mathrm{m}, \mathrm{C}^{15} \mathrm{H}\right), 7.86\left(2 \mathrm{H}, \mathrm{d},{ }^{3} J_{H H}=4.4 \mathrm{~Hz}, \mathrm{C}^{14} \mathrm{H}\right), 7.31\left(2 \mathrm{H}, \mathrm{dd},{ }^{3} J_{H H}=7.3\right.$ $\left.\mathrm{Hz},{ }^{3} J_{H H}=4.4 \mathrm{~Hz}, \mathrm{C}^{13} \mathrm{H}\right), 6.74\left(8 \mathrm{H}, \mathrm{s}, \mathrm{C}^{6} \mathrm{H}\right), 6.64\left(2 \mathrm{H}, \mathrm{m}, \mathrm{C}^{12} \mathrm{H}\right), 4.59$ $\left(4 \mathrm{H}\right.$, br.d $\left.,{ }^{2} J_{H H}=12.4 \mathrm{~Hz}, \mathrm{C}^{1} \mathrm{H}_{\mathrm{B}}\right), 4.16\left(2 \mathrm{H}\right.$, br.d, $\left.{ }^{2} J_{H H}=13.2 \mathrm{~Hz}, \mathrm{C}^{10} \mathrm{H}_{\mathrm{B}}\right)$, 
$3.82\left(2 \mathrm{H}\right.$, br.d, $\left.{ }^{2} \mathrm{~J}_{H H}=13.2 \mathrm{~Hz}, \mathrm{C}^{10} \mathrm{H}_{\mathrm{A}}\right), 2.83\left(4 \mathrm{H}, \mathrm{dm},{ }^{2} J_{H H}=12.4 \mathrm{~Hz}\right.$, $\left.\mathrm{C}^{1} \mathrm{H}_{\mathrm{A}}\right), 2.59$ (24H, s, C $\left.{ }^{8} \mathrm{H}\right), 2.14\left(12 \mathrm{H}, \mathrm{s}, \mathrm{C}^{9} \mathrm{H}\right), 1.86-2.28(12 \mathrm{H}, \mathrm{m}$, $\left.\mathrm{C}^{2} \mathrm{H}+\mathrm{C}^{3} \mathrm{H}\right) .{ }^{31} \mathrm{P}$ NMR $\left(\mathrm{C}_{6} \mathrm{D}_{6}, 303 \mathrm{~K}\right) \delta_{\mathrm{p}} \mathrm{ppm}:-41.70(\mathrm{~s})$.

(RSSR)-1,9-Di-[(pyridine-4'-yl)methyl]-3, 7,11,15tetramesityl-1,9-diaza-3,7,11,15-tetraphosphacyclohexadecane (13) was obtained like 6 from 1,3-bis(mesitylphosphino)propane (1.08 g, $3.13 \mathrm{mmol})$, paraformaldehyde $(0.18 \mathrm{~g}, 6.0 \mathrm{mmol})$ and 4-(aminomethyl)pyridine (0.34 g, $3.15 \mathrm{mmol})$. Yield of $\mathbf{1 3} 1.07$ g (72 \%); m.p. $187-189{ }^{\circ} \mathrm{C}$. Found: C 72.90; H 8.20; N 6.11; P $12.71 \% . \mathrm{C}_{58} \mathrm{H}_{76} \mathrm{P}_{4} \mathrm{~N}_{4}$ [953.15] requires $\mathrm{C} 73.09 ; \mathrm{H} 8.04 ; \mathrm{N}$ 5.88; $\mathrm{P}$ $13.00 \% .{ }^{1} \mathrm{H}$ NMR $\left(\mathrm{C}_{6} \mathrm{D}_{6}, 303 \mathrm{~K}\right) \delta_{\mathrm{H}} \mathrm{ppm}: 8.48\left(4 \mathrm{H}, \mathrm{d},{ }^{3} J_{H H}=4.4 \mathrm{~Hz}\right.$, $\left.\mathrm{C}^{13} \mathrm{H}\right), 7.21\left(4 \mathrm{H}, \mathrm{d},{ }^{3} J_{H H}=4.4 \mathrm{~Hz}, \mathrm{C}^{12} \mathrm{H}\right), 6.82\left(8 \mathrm{H}, \mathrm{s}, \mathrm{C}^{6} \mathrm{H}\right), 4.42(4 \mathrm{H}$, br.d, $\left.{ }^{2} J_{H H}=12.4 \mathrm{~Hz}, \mathrm{C}^{1} \mathrm{H}_{\mathrm{B}}\right), 3.94\left(2 \mathrm{H}\right.$, br.d, $\left.{ }^{2} J_{H H}=13.2 \mathrm{~Hz}, \mathrm{C}^{10} \mathrm{H}_{\mathrm{B}}\right)$, $3.12\left(2 \mathrm{H}\right.$, br.d, $\left.{ }^{2} J_{H H}=13.2 \mathrm{~Hz}, \mathrm{C}^{10} \mathrm{H}_{\mathrm{A}}\right), 2.66\left(4 \mathrm{H}, \mathrm{m}, \mathrm{C}^{1} \mathrm{H}_{\mathrm{A}}\right), 2.48$ $\left(24 \mathrm{H}, \mathrm{s}, \mathrm{C}^{8} \mathrm{H}\right), 2.23\left(12 \mathrm{H}, \mathrm{s}, \mathrm{C}^{9} \mathrm{H}\right), 1.91-2.38\left(12 \mathrm{H}, \mathrm{m}, \mathrm{C}^{2} \mathrm{H}+\mathrm{C}^{3} \mathrm{H}\right)$. ${ }^{13} \mathrm{C}$ NMR $\left(\mathrm{CDCl}_{3}, 303 \mathrm{~K}\right) \delta_{\mathrm{C}} \mathrm{ppm}: 149.47\left(\mathrm{~s}, \mathrm{C}^{13}\right), 149.15\left(\mathrm{~s}, \mathrm{C}^{13}\right)$, $144.41\left(\mathrm{~d},{ }^{2} J_{C P}=15.2 \mathrm{~Hz}, \mathrm{C}^{5}\right), 139.06\left(\mathrm{~s}, \mathrm{C}^{7}\right), 130.18\left(\mathrm{~d},{ }^{1} J_{C P}=19.2\right.$ $\left.\mathrm{Hz}, \mathrm{C}^{4}\right), 129.56\left(\mathrm{~s}, \mathrm{C}^{11}\right), 121.27-123.55\left(\mathrm{~m}, \mathrm{C}^{6}+\mathrm{C}^{12}\right), 56.59-59.94$ (br.m, $\left.\mathrm{C}^{1}+\mathrm{C}^{10}\right), 27.98-29.31\left(\mathrm{~m}, \mathrm{C}^{2}+\mathrm{C}^{3}\right), 23.57\left(\mathrm{~d},{ }^{3} J_{C P}=18.9 \mathrm{~Hz}\right.$, $\left.\mathrm{C}^{8}\right), 20.85\left(\mathrm{~s}, \mathrm{C}^{9}\right) .{ }^{31} \mathrm{P}$ NMR $\left(\mathrm{C}_{6} \mathrm{D}_{6}, 303 \mathrm{~K}\right) \delta_{\mathrm{p}} \mathrm{ppm}:-41.50(\mathrm{~s})$.

(RSSR)-1,9-Di-[2'-(pyridine-2'"-yl) ethyl]-3, 7,11,15-tetramesityl-1,9-diaza-3,7,11,15-tetraphosphacyclohexadecane (14) was obtained like 6 from 1,3-bis(mesitylphosphino)propane $(0.5$ g, $1.45 \mathrm{mmol})$, paraformaldehyde $(0.09 \mathrm{~g}, 3.0 \mathrm{mmol})$ and 2-(2'aminoethyl)pyridine ( $0.18 \mathrm{~g}, 1.47 \mathrm{mmol})$. Yield of $140.29 \mathrm{~g}$ (41 \%); m.p. 194-196 ${ }^{\circ}$ C. Found: C 73.08; H 8.33; N 5.98; P $12.54 \%$. $\mathrm{C}_{60} \mathrm{H}_{80} \mathrm{P}_{4} \mathrm{~N}_{4}$ [981.20] requires $\mathrm{C} 73.45 ; \mathrm{H} 8.22 ; \mathrm{N} 5.71 ; \mathrm{P} 12.63 \%$. ${ }^{1} \mathrm{H}$ NMR $\left(\mathrm{C}_{6} \mathrm{D}_{6}, 303 \mathrm{~K}\right) \delta_{\mathrm{H}} \mathrm{ppm}: 8.46\left(2 \mathrm{H}, \mathrm{d},{ }^{3} \mathrm{~J}_{H H}=3.6 \mathrm{~Hz}, \mathrm{C}^{16} \mathrm{H}\right)$, $7.10\left(2 \mathrm{H}, \mathrm{m}, \mathrm{C}^{14} \mathrm{H}\right), 6.86\left(2 \mathrm{H}, \mathrm{d},{ }^{3} J_{H H}=7.7 \mathrm{~Hz}, \mathrm{C}^{13} \mathrm{H}\right), 6.75(8 \mathrm{H}, \mathrm{s}$, $\left.\mathrm{C}^{6} \mathrm{H}\right), 6.64\left(2 \mathrm{H}, \mathrm{m}, \mathrm{C}^{15} \mathrm{H}\right), 4.12\left(4 \mathrm{H}\right.$, br.d, $\left.{ }^{2} \mathrm{~J}_{H H}=12.4 \mathrm{~Hz}, \mathrm{C}^{1} \mathrm{H}_{\mathrm{B}}\right), 3.57$ $\left(2 \mathrm{H}, \mathrm{m}, \mathrm{C}^{10} \mathrm{H}_{\mathrm{B}}\right), 3.05\left(2 \mathrm{H}, \mathrm{m}, \mathrm{C}^{10} \mathrm{H}_{\mathrm{A}}\right), 2.77$ (4H, br.m., $\left.\mathrm{C}^{1} \mathrm{H}_{\mathrm{A}}\right), 2.62$ (4H, m, C $\left.{ }^{11} \mathrm{H}\right), 2.59$ (24H, s, C $\left.{ }^{8} \mathrm{H}\right), 2.08\left(12 \mathrm{H}, \mathrm{s}, \mathrm{C}^{9} \mathrm{H}\right), 1.80-2.45$ $\left(12 \mathrm{H}, \mathrm{m}, \mathrm{C}^{2} \mathrm{H}+\mathrm{C}^{3} \mathrm{H}\right) .{ }^{13} \mathrm{C}$ NMR $\left(\mathrm{CDCl}_{3}, 303 \mathrm{~K}\right) \delta_{\mathrm{C}}$ ppm: 149.90 $\left(\mathrm{s}, \mathrm{C}^{16}\right), 149.58\left(\mathrm{~s}, \mathrm{C}^{12}\right), 143.96\left(\mathrm{~d},{ }^{2} J_{C P}=15.1 \mathrm{~Hz}, \mathrm{C}^{5}\right), 138.89(\mathrm{~s}$, $\left.\mathrm{C}^{7}\right), 130.32\left(\mathrm{~d},{ }^{1} J_{C P}=19.2 \mathrm{~Hz}, \mathrm{C}^{4}\right), 130.18\left(\mathrm{~s}, \mathrm{C}^{14}\right), 121.27-124.07$ $\left(\mathrm{m}, \mathrm{C}^{6}+\mathrm{C}^{13}+\mathrm{C}^{15}\right)$, 55.89-59.64 (br.m, $\left.\mathrm{C}^{1}+\mathrm{C}^{10}\right)$, 28.06-29.01 (m, $\left.\mathrm{C}^{2}+\mathrm{C}^{3}+\mathrm{C}^{11}\right), 23.37\left(\mathrm{~d},{ }^{3} \mathrm{~J}_{C P}=18.9 \mathrm{~Hz}, \mathrm{C}^{8}\right), 20.69\left(\mathrm{~s}, \mathrm{C}^{9}\right) .{ }^{31} \mathrm{P}$ NMR $\left(\mathrm{C}_{6} \mathrm{D}_{6}, 303 \mathrm{~K}\right) \delta_{\mathrm{P}} \mathrm{ppm}:-41.80(\mathrm{~s})$.

\section{X-Ray Crystallography}

Data for 6, 7, 9, 10 were collected at 293(2) K on a Bruker Smart APEX II CCD diffractometer with graphite monochromated MoK $\alpha$ radiation $(\lambda=0.71073 \AA)$ and $\omega$-scan rotation. Data collection and reduction were performed using the APEX2 and SAINT ${ }^{[20]}$ software. Absorption corrections were performed using the SADABS ${ }^{[1]}$ program. All the structures were solved by direct methods and refined by full-matrix least-squares methods against $\mathrm{F}^{2}$ with the SHELX ${ }^{[22]}$ software package. All non-H atoms were refined anisotropically. All $\mathrm{H}$ atoms were fixed at calculated positions and refined with the use of a riding model. The data for 11 were collected on a Siemens CCD diffractometer (SMART) ${ }^{[23]}$ using Mo- $\mathrm{K}_{\alpha}$ radiation $(\lambda=0.71073 \AA)$ and $\omega$-scan rotation. Data reduction was performed with SAINT ${ }^{[24]}$ including the program SADABS $^{[21]}$ for empirical absorption correction. The structure 11 was solved by direct methods (SIR-92) ${ }^{[25]}$ and the refinement of all non-hydrogen atoms was performed with SHELXL-97. ${ }^{[22]}$ For 11 all $\mathrm{H}$ atoms were located on difference Fourier maps calculated at the final stage of the structure refinement. Crystallographic data for 6, 7, 9-11 are given in Table 1.

Table 1. Crystallographic data for 6, 7, 9, 10, 11 .

\begin{tabular}{|c|c|c|c|c|c|}
\hline & 6 & 7 & 9 & 10 & 11 \\
\hline Formula & $\mathrm{C}_{62} \mathrm{H}_{82} \mathrm{~N}_{2} \mathrm{P}_{4} \cdot \mathrm{C}_{6} \mathrm{H}_{6}$ & $\mathrm{C}_{68} \mathrm{H}_{94} \mathrm{~N}_{2} \mathrm{P}_{4}$ & $\mathrm{C}_{64} \mathrm{H}_{86} \mathrm{~N}_{2} \mathrm{P}_{4}$ & $\mathrm{C}_{62} \mathrm{H}_{78} \mathrm{~N}_{2} \mathrm{O}_{4} \mathrm{P}_{4}$ & $\mathrm{C}_{64} \mathrm{H}_{82} \mathrm{~N}_{4} \mathrm{P}_{4}$ \\
\hline M & 1057.28 & 1063.33 & 1007.23 & 1039.14 & 1031.22 \\
\hline $\mathrm{T}[\mathrm{K}]$ & 293(2) & 293(2) & 293(2) & 293(2) & 213(2) K \\
\hline$\lambda[\AA]$ & 0.71073 & 0.71073 & 0.71073 & 0.71073 & 0.71073 \\
\hline Crystal system & Triclinic & Triclinic & Triclinic & Triclinic & Triclinic \\
\hline Space group & P-1 & $\mathrm{P}-1$ & P-1 & P-1 & P-1 \\
\hline $\mathrm{a}[\AA]$ & $8.9220(5)$ & $11.629(7)$ & $10.3863(8)$ & $11.038(5)$ & $8.7619(5)$ \\
\hline $\mathrm{b}[\AA]$ & $12.8404(7)$ & $11.826(7)$ & $11.8295(9)$ & $12.034(5)$ & $12.9314(8)$ \\
\hline $\mathrm{c}[\AA]$ & $15.1050(9)$ & $13.372(8)$ & $13.4825(10)$ & $12.908(5)$ & $14.9375(9)$ \\
\hline$\alpha\left[^{\circ}\right]$ & $68.473(1)$ & $73.478(7)$ & $70.171(1)$ & $103.581(5)$ & $65.791(1)$ \\
\hline$\beta\left[^{\circ}\right]$ & $80.804(1)$ & $68.639(7)$ & $79.589(1)$ & $103.369(5)$ & $79.679(1)$ \\
\hline$\gamma\left[^{\circ}\right]$ & $75.654(1)$ & $80.910(8)$ & $85.481(1)$ & $106.863(5)$ & $74.680(1)$ \\
\hline $\mathrm{V}\left[\AA^{3}\right]$ & $1554.99(15)$ & 1638.9(17) & $1532.4(2)$ & $1510.1(11)$ & $1483.9(2)$ \\
\hline Z & 1 & 1 & 1 & 1 & 1 \\
\hline $\begin{array}{l}\text { Reflections } \\
\text { collected }\end{array}$ & 17035 & 16761 & 11660 & 13208 & 9252 \\
\hline $\begin{array}{l}\text { Independent } \\
\text { reflections }\end{array}$ & 6733 & 6416 & 5937 & 6944 & 6828 \\
\hline $\begin{array}{l}\text { Reflection with } I \\
\quad \geq 2 \sigma\end{array}$ & 4643 & 3019 & 4451 & 1217 & 5683 \\
\hline$R_{i n t}$ & 0.029 & 0.0748 & 0.0157 & 0.3679 & 0.0096 \\
\hline $\operatorname{GOF}\left(F^{2}\right)$ & 1.01 & 0.954 & 1.018 & 0.751 & 1.013 \\
\hline$\rho_{\text {calcd. }}\left[\mathrm{g} . \mathrm{cm}^{-3}\right]$ & 1.129 & 1.077 & 1.091 & 1.143 & 1.154 \\
\hline$\mu\left[\mathrm{mm}^{-1}\right]$ & 0.162 & 0.154 & 0.161 & 0.17 & 0.169 \\
\hline $\mathrm{R}\left(F_{\mathrm{o}}\right),[I>2 \sigma(I)]$ & 0.0469 & 0.0628 & 0.0630 & 0.0990 & 0.0373 \\
\hline $\mathrm{R}_{w}\left(F \mathrm{O}^{2}\right)$ & 0.1318 & 0.1332 & 0.1817 & 0.1143 & 0.0991 \\
\hline $\begin{array}{c}\Delta \rho(\max . / \min .) \\
{\left[\mathrm{e} \AA^{-3}\right]}\end{array}$ & $0.32 /-0.20$ & $0.324 /-0.16$ & $0.708 /-0.422$ & $0.238 /-0.204$ & $0.303 /-0.193$ \\
\hline
\end{tabular}


CCDC 1006643 (6), 1006641 (7), 1006642 (9), 1006640 (10) and 1004851 (11) contain the supplementary crystallographic data for this paper. These data can be obtained free of charge from The Cambridge Crystallographic Data Centre via www.ccdc.cam.ac.uk/ data_request/cif.

\section{Results and Discussion}

It has been established that 1,3-bis(phenylphosphino) propane and 1,3-bis(mesitylphosphino)propane have a similar reactivity in the desired Mannich-type condensation. [13-16] Because the last diphosphine with mesityl groups on phosphorus atoms is noticeably more stable to air oxidation and smells less repulsive we have used it as a starting compound for the developing of the self-assembly approach to the 16-membered P,N-containing macrocycles.

It had been shown previously that desired self-assembly condensation of 16-membered macrocycles required relatively basic primary amines. One of the most interesting features of aminomethylphosphine chemistry is the possibi lity to obtain a wide set of functionalized phosphine ligands on the basis of the various available primary amines. It was shown that self-assembly processes occurred with wide variety of primary amines to give crystalline 1,9-diaza3,7,11,15-tetraphosphacyclohexadecanes 6-14 (Scheme 2) in good yields (33-72\%). The practically pure desired macrocycles 6-14 crystallized spontaneously from the reaction mixtures in the course of the condensations and were isolated by filtration. The reaction time depended on the nature of primary amines, the temperature and the used solvents. We also showed that it was better to perform the condensation in two steps. At the first step 1,3-bis(mesitylphosphino)propane reacted with formaldehyde at elevated temperature $\left(50-80{ }^{\circ} \mathrm{C}\right)$ or with $30 \%$ formalin in ethanol at room temperature to give 1,3-bis[hydroxymethyl(mesityl)phosphino] propane. On the second step the obtained diol interacted with primary amines in DMF (as a rule) or toluene at $75-110{ }^{\circ} \mathrm{C}$ for few hours (the heating of reaction mixture overnight was the most convenient). If the initial reagents were combined all together in one reaction vessel simultaneously the reaction time was noticeably longer and the total yields of title macrocycles were $\sim 10 \%$ less than expected.

The reactions were performed in the concentration range $0.1-0.5 \mathrm{M}$ at elevated temperatures $\left(75-110^{\circ} \mathrm{C}\right)$ and in the absence of the templating reagents. The NMR monitoring of the reaction mixtures showed that the reactions proceeded with the formation of plenty of intermediates and the reaction mixtures were enriched by the isolated products only at the final stage, as in the case of macrocyclizations described above. ${ }^{[14-16]}$ Thus, we have again met the phenomenon of the covalent self-assembly.

It should be mentioned that 2-phenylethyl- and 3-phenylpropylamines which were more basic than previously reported benzylamines gave $\mathrm{P}, \mathrm{N}$-corands $\mathbf{8}$ and 9 without formation of side-products usually expected for the condensation of highly basic amines. ${ }^{[14]}$ The presence of functional groups in the starting amines namely 1,3-dioxolyl one in the case of the synthesis of corand $\mathbf{1 0}$ and electronwithdrawing pyridyl-containing substituents in the case of synthesis of corands 11-14 did not change the outcome reaction too.

$\mathrm{P}, \mathrm{N}$-containing corands 6-14 were obtained in a pure form as air-stable white crystalline solids. In the NMR spectra of freshly dissolved compounds 6-14 only one set of signals attributed to one stereoisomer was observed. Signals of core ring protons were similar for all obtained macrocycles and resembled the picture characteristic for corands $\mathbf{1 - 4},{ }^{[15,16]}$ including unusual high-field shifted $\mathrm{H}^{\mathrm{A}}$ signal of $\mathrm{P}-\mathrm{CH}^{\mathrm{A}}-\mathrm{N}$ fragment at $2.66-2.83 \mathrm{ppm}(3.2-3.6 \mathrm{ppm}$ is an ordinary value of chemical shifts of high-field signal of $\mathrm{P}-\mathrm{CH}_{2}-\mathrm{N}$ cyclic fragments). The last observation could be regarded as a characteristic of a macrocycle formation. The spectral

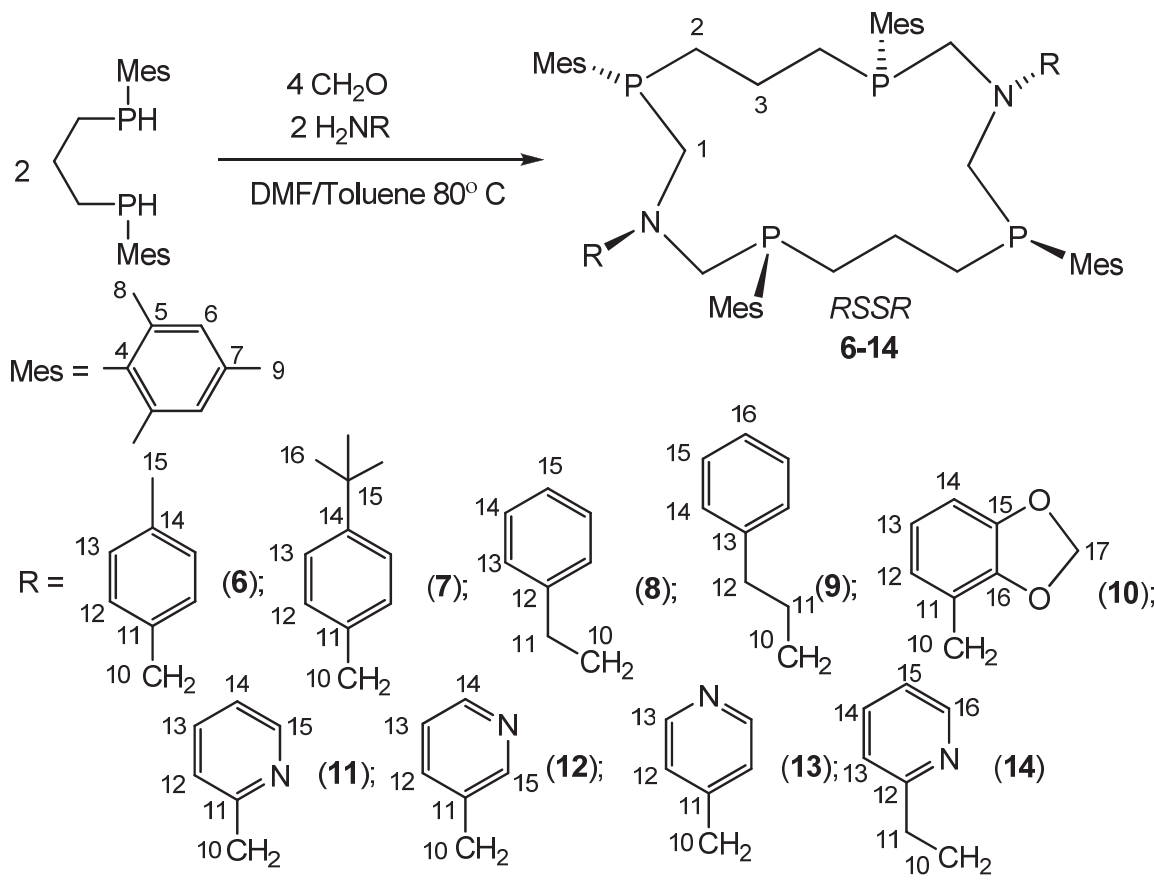

Scheme 2. 
data were consisted with the formation of only one RSSRstereoisomer of macrocycles 6-14. The macrocycles 6-14 contain four phosphorus asymmetric centers. The starting 1,3-bis(mesitylphosphino)propane exists as a mixture of rac- and meso-stereoisomers, and it must lead to the formation of five possible diastereoisomers. Therefore, in the course of the reaction, not only the mutual recognition of the intermediate stereoisomers takes place but also the inversion of the starting diphosphine configuration proceeds. ${ }^{[14]}$

Single-crystal X-ray analysis established the structures of the $R S S R$ stereoisomers of 6, 7, 9, 10 and 11 (Figures 1-5).

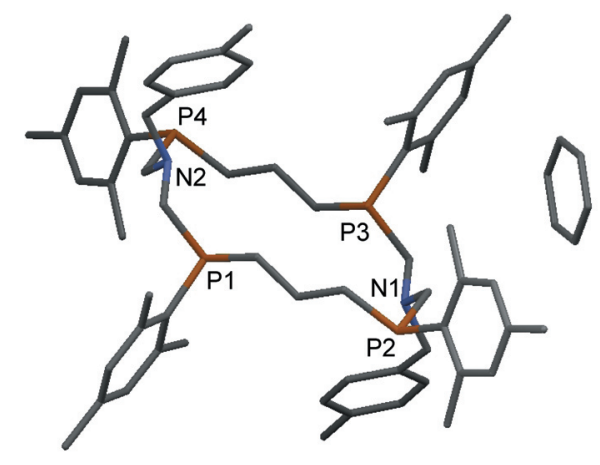

Figure 1. The molecular structure of the macrocycle 6 with solvate benzene molecule (hydrogen atoms are omitted for clarity).

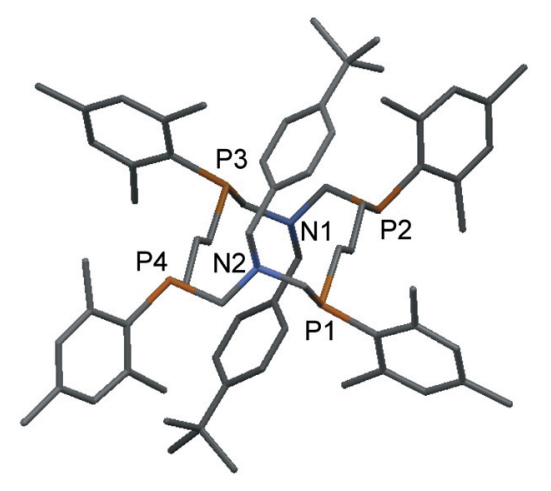

Figure 2. The molecular structure of the macrocycle 7 (hydrogen atoms are omitted for clarity).

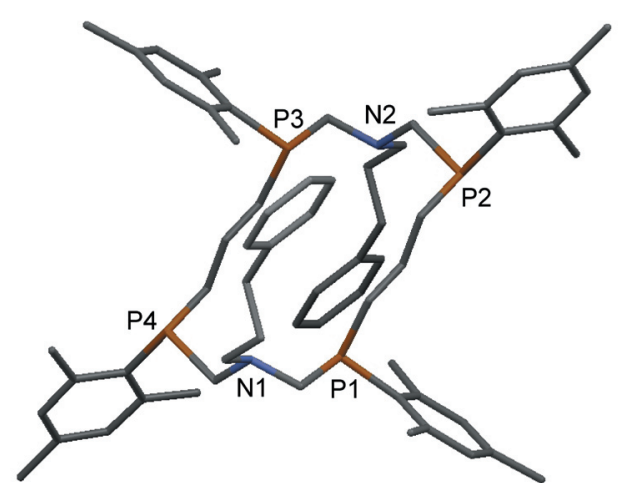

Figure 3. The molecular structure of the macrocycle 9 (hydrogen atoms are omitted for clarity).

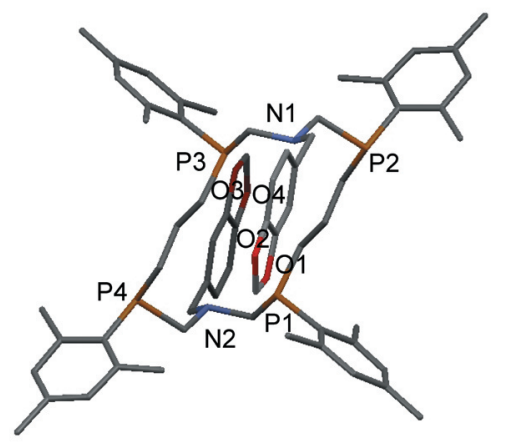

Figure 4. The molecular structure of the macrocycle 10 (hydrogen atoms are omitted for clarity).

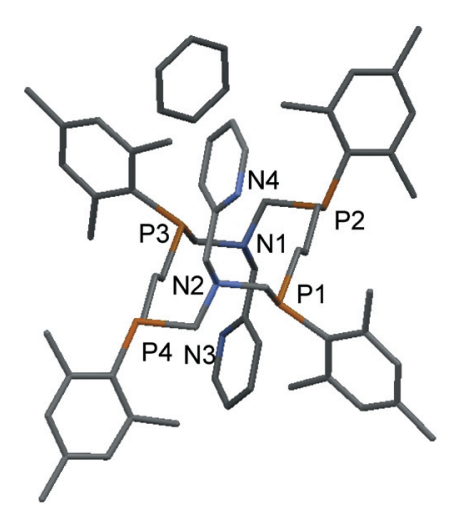

Figure 5. The molecular structure of the macrocycle $\mathbf{1 1}$ with solvate benzene molecule (hydrogen atoms are omitted for clarity).

In total the structures of the core-rings were similar to that of previously described corands $\mathbf{1 - 4} .^{[15,16]}$ It should be noted that in all the crystals $\mathbf{6 , 7 , 9 , 1 0}$ and $\mathbf{1 1}$, the molecules are in a special position of the center of inversion, i.e. 16-membered heterocycles have centrosymmetric conformation, which may be described as a "distorted crown". As a result, opposite phosphorus atoms have the different configuration, and the molecule as a whole constitutes a mesoform. Two centrosymmetric $-\mathrm{PCH}_{2} \mathrm{NCH}_{2} \mathrm{P}$ - fragments demonstrate twist-conformation with inequivalent methylene groups and the nitrogen lone pairs looking just into the molecular cavity. Perhaps, that unusual for ordinary heterocyclic aminomethylphosphines conformation is kept in solution and it is the reason of unusual chemical shifts of methylene protons.

Four phosphorus atoms of all macrocycles are situated in one plane and form a parallelogram with narrow P1 $\cdots \mathrm{P} 2$ and $\mathrm{P} 2 \cdots \mathrm{P} 3$ distances distributions (dP1 $\cdots \mathrm{P} 2$ are in the range from $5.55 \AA$ for 7 to $5.62 \AA$ for $\mathbf{1 1}$ and $\mathrm{dP} 2 \cdots \mathrm{P} 3$ are in the range $4.45 \AA$ for $\mathbf{1 0}$ to $4.49 \AA$ for $\mathbf{6}, 7$ and 11). However the shortest diagonal distances P1 $\cdots \mathrm{P} 3$ are noticeably elongated with the increasing of the bulkiness of substituents on nitrogen atoms $(4.76 \AA$ (6), $4.94 \AA$ (10), $4.98 \AA$ (11), $5.48 \AA(9)$ and $5.83 \AA(7))$. The longest diagonal P2 $\cdots \mathrm{P} 4$ distances are shortened in nearly the same row $(8.96 \AA$ (6), $8.87 \AA$ (11), $8.81 \AA$ (10), $8.50 \AA$ (9) and $8.24 \AA$ (7)). So the compounds 7 and 9 have the largest sectional areas of 

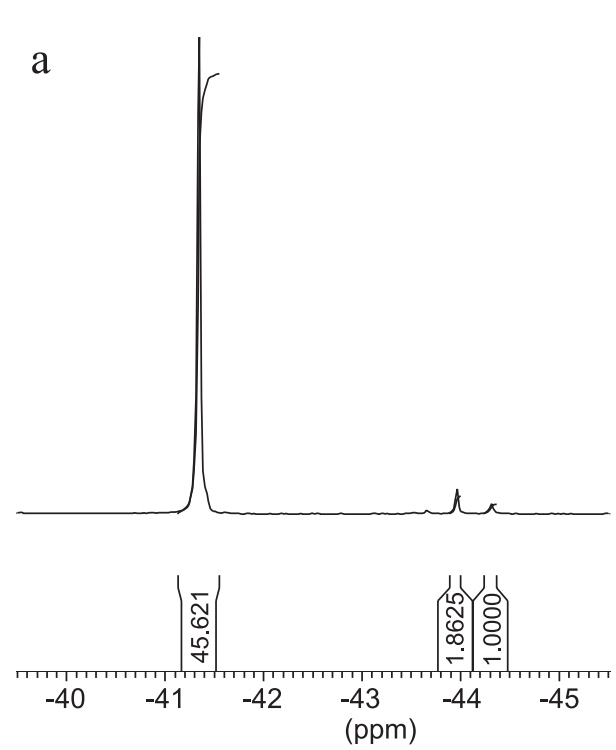
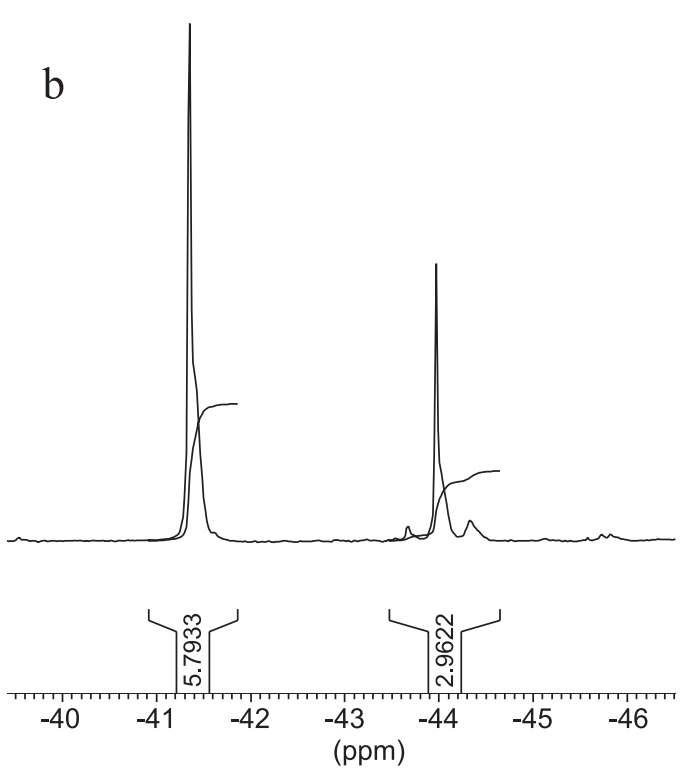

Figure 6. The ${ }^{31} \mathrm{P}$ NMR spectrum of $6\left(\mathrm{C}_{6} \mathrm{D}_{6}\right)$ after $2 \mathrm{~h}(\mathrm{a})$ and the ${ }^{31} \mathrm{P}$ NMR spectrum of the equilibrated isomeric mixture of $\mathbf{6}\left(\mathrm{C}_{6} \mathrm{D}_{6}\right)$ after 1 week (b).

macrocyclic cavity and at the same time in these compounds the cavity spaces are isolated from outer space by bulky pseudoaxial substituents on nitrogen atoms situated just over and under the cavities. The structure of macrocycle $\mathbf{1 1}$ is very similar to that of $\mathbf{2}^{[15]}$ and $\mathbf{6}$. The geometry of the molecule is shown in Figure 5. In the solid state the pyridyl groups are located over and under the cavity of the macrocycle, and their nitrogen atoms are looking outward the molecular cavity and are remote from the phosphorus atoms. However in the solutions the rotation around C-N and $\mathrm{C}-\mathrm{C}$ bonds of exocyclic pyridylmethyl fragments is possible and as a result the positions of phosphorus and nitrogen donor centers may become more close and favorable for the formation of N,P-chelate complexes with various transition metals.

It has been shown that soon after the dissolving the additional set of signals appeared in the ${ }^{31} \mathrm{P}$ NMR spectra of macrocycles 1, $\mathbf{4}^{[26]}$ and 6-12 (Figure 6). The equilibrium was established within a few days. The chemical shifts of the appearing signals (for example -44.1, -44.4 и $-44.7 \mathrm{ppm}$ for $\mathbf{6}$ (Figure 6b)) were similar for all macrocyclic products. We suppose that a set of additional signals belongs to other stereoisomers of macrocyclic tetraphosphines. The remar-kable fast phosphorus epimerization was recently shown for the relevant heterocycles, namely 1-aza-3,6-diphosphacycloheptanes. ${ }^{[27]}$ However we could not exclude the processes of corresponding ring narrowing (dissociation of macrocycles into two 8-membered 1-aza3,7-diphosphacyclooctanes). It should be mentioned that the initial signal was still predominant (Figure 6b), demonstrating the thermodynamic preference of the starting isomer of the macrocycle. The removal of the solvent and the recrystallization led to the pure macrocycles 6-14 in very good yields.

So, the general character of the covalent selfassembly of corands with 1,9-diaza-3,7,11,15-tetraphosphacyclohexadecane core ring was demonstrated.

\section{Conclusions}

The Mannich-type condensations in three component systems: 1,3-bis(mesitylphosphino)propane-formaldehydeprimary amines containing aryl (or hetaryl) group which is separated from amino group by $\mathrm{C}_{1}-\mathrm{C}_{3}$ alkylene spacer - proceed as the covalent self-assembly processes to give 1,9-di(aralkyl)-3,7,11,15-tetramesityl-1,9-diaza3,7,11,15-tetraphosphacyclohexadecanes in good yields. The macrocyclization was stereoselective and led to $R S S R$-stereoisomers of these 16-membered P,N-containing corands. The successful synthesis of macrocycles on the basis of the wide variety of starting diamines with electrondonating and electron-withdrawing groups and various lengths of alkylene spacers indicates the general character of the covalent self-assembly of 1,9-diaza-3,7,11,15tetraphosphacyclohexadecanes and the effectiveness of the described synthetic approach. In the solid state $R S S R$-stereoisomers of all studied corands have similar centrosymmetric conformations which may be described as a "distorted crown" with the different configurations of opposite phosphorus atoms so that the molecule as a whole constitute a mesoform. The substituents on nitrogen atoms are situated just over and under the cavities. In the solutions $R S S R$-stereoisomers of 1,9-diaza-3,7,11,15-tetraphosphacyclohexadecanes undergo the partial transformation which is most likely the stereoconversion into other stereoisomers of corresponding macrocyclic tetraphoshines, but the starting RSSR-isomers remain predominant as the most thermodynamically stable ones.

Acknowledgements. This work was supported by the Russian Foundation for Basic Research (grants 10-0300380_a, 12-03-97083-r_povolzhie_a), a President of Russia grant for the support of leading scientific schools (grant NSh-4428.2014.3), Ministry of Education and Science of Russian Federation (state contract 8432). 


\section{References}

1. Steed J.W., Atwood J.L. Supramolecular Chemistry, John Wiley \& Sons, Chichester, 2000.

2. Macrocyclic Chemistry (Gloe K., Ed.), Springer, 2005.

3. Chapman R.G., Sherman J.C. Tetrahedron 1997, 53, 1591115945

4. Caricato M., Coluccini C., Dondi D., Van der Griend D.A., Pasini D. Org. Biomol. Chem. 2010, 8, 3272-3280

5. Core Concepts in Supramolecular Chemistry and Nanochemistry (Steed J.W., Turner D.R., Wallace K.J., Eds.). Wiley-VCH, Weinhein, 2007.

6. Lehn J.-M. Supramolecular Chemistry: Concepts and Perspectives, Wiley-VCH, Weinheim, 1995.

7. Nanomaterials Chemistry: Recent Developments and New Directions (Rao C.N.R., Müller A., Cheetham A.K., Eds.). Wiley-VCH, Weinheim, 2007.

8. Edwards P.G, Ingold F., Liyanage S.S., Newman P.D., Wong W-K., Chen Y. Eur. J. Inorg Chem. 2001, 2865-2869.

9. Bauer I., Gruner M., Goutal S., Habicher W.D. Chem. Eur. J. 2004, 10, 4011-4016.

10. Philp D., Stoddart J.F. Angew. Chem., Int. Ed. 1996, 35, 11541196

11. Greig L.M., Philp D. Chem. Soc. Rev. 2001, 30, 287-302.

12. Otto S., Furlan R.L.E., Sanders J.K.M. Drug Discovery Today 2002, 7, 117-125.

13. Karasik A.A., Sinyashin O.G. Phosphorus Based Macrocyclic Ligands: Synthesis and Applications. In: Catalysis by Metal Complexes. Vol. 37. Phosphorus Compounds: Advanced Tools in Catalysis and Material Sciences (Gonsalvi L., Peruzzini M., Eds). Dordrecht: Springer Netherlands, 2011, Ch. 12, p. 377448.

14. Karasik A.A., Balueva A.S., Sinyashin O.G. C. R. Chimie 2010, 13, 1151-1167.
15. Naumov R.N., Karasik A.A., Sinyashin O.G., Lönnecke P., Hey-Hawkins E. Dalton Trans. 2004, 357-358.

16. Naumov R.N., Karasik A.A., Kanunnikov K.B., Kozlov A.V., Latypov Sh.K., Domasevich K.V., Hey-Hawkins E., Sinyashin O.G. Mendeleev Commun. 2008, 18, 80-81.

17. Naumov R.N., Kozlov A.V., Kanunnikov K.B., Gómez-Ruiz S., Hey-Hawkins E., Latypov Sh.K., Karasik A.A., Sinyashin O.G. Tetrahedron Lett. 2010, 51, 1034-1037.

18. Swor C.D., Tyler D.R. Coord. Chem. Rev. 2011, 255, 28602881.

19. Karasik A.A., Naumov R.N., Spiridonova Y.S., Sinyashin O.G., Lonnecke P., Hey-Hawkins E. Z. Anorg. Allg. Chem. 2007, 633, 205-210.

20. APEX2 (Version 2.1), SAINTPlus. Data Reduction and Correction Program (Version 7.31A, Bruker Advansed X-Ray Solutions. BrukerAXS Inc., Madison, Wisconsin, USA, 2006.

21. Sheldrick G.M., SADABS, Program for Scaling and Correction of Area-Detector Data, Göttingen, 1997.

22. Sheldrick G.M. A Short History of SHELX. Acta Cryst. 2008, A64, 112-122.

23. SMART: Area-Detector Software Package. Siemens Industrial Automation, Inc.: Madison, WI, 1993.

24. SAINT: Area-Detector Integration Software. Version 6.01. Siemens Industrial Automation, Inc.: Madison, WI, 1999.

25. Altomare A., Cascarano G., Giacovazzo C., Guagliardi A. J. Appl. Crystallogr. 1994, 27, 435.

26. Naumov R.N., Karasik A.A., Kozlov A.V., Latypov Sh.K., Krivolapov D.B., Dobrynin A.B., Litvinov I.A., Kataeva O.N., Lönnecke P., Hey-Hawkins E., Sinyashin O.G. Phosphorus, Sulfur, Silicon Relat. Elem. 2008, 183, 456-459.

27. Musina E.I., Karasik A.A., Balueva A.S., Strelnik I.D., Fesenko T.I., Dobrynin A.B., Gerasimova T.P., Katsyuba S.A., Kataeva O.N., Lönnecke P., Hey-Hawkins E., Sinyashin O.G. Eur. J. Inorg. Chem. 2012, 1857-1866. 